\title{
Anterior skull base reconstruction using an anterolateral thigh free flap
}

\author{
Shin Hyun Kim ${ }^{1,2}$, \\ Won Jai Lee ${ }^{1,2}$, \\ Jong Hee Chang', \\ Joo Hyung Moon ${ }^{3}$, \\ Seok Gu Kang ${ }^{3}$, \\ Chang Hoon Kim ${ }^{4}$, \\ Jong Won Hong ${ }^{1,2}$ \\ ${ }^{1}$ Department of Plastic and \\ Reconstructive Surgery, ${ }^{2}$ Institute for \\ Human Tissue Restoration, and \\ Departments of ${ }^{3}$ Neurosurgery and \\ ${ }^{4}$ Otorhinolaryngology, Yonsei University \\ College of Medicine, Seoul, Korea
}

\begin{abstract}
Background: Galeal or temporalis muscle flaps have been traditionally used to reconstruct skull base defects after tumor removal. Unfortunately, these flaps do not provide sufficient vascularized tissue for a dural seal in extensive defects. This study describes the successful coverage of large skull base defects using anterolateral thigh (ALT) free flaps.

Methods: This retrospective study included five patients who underwent skull base surgery between June 2018 and June 2021. Reconstruction was performed using an ALT free flap to cover defects that included the intracranial space and extended to the frontal sinus and cribriform plate. Results: There were no major complications, such as ascending infections or cerebrospinal leakage. Postoperative magnetic resonance imaging showed that the flaps were well-maintained in all patients.

Conclusion: Successful reconstruction was performed using ALT free flaps for large anterior skull base defects. In conclusion, the ALT free flap is an effective option for preventing communication between the nasal cavity and the intracranial space.
\end{abstract}

Abbreviations: ALT, anterolateral thigh; MRI, magnetic resonance imaging; STA, superior temporal artery; STV, superior temporal vein.

Keywords: Free tissue flaps / Reconstructive surgery / Temporal artery

\section{INTRODUCTION}

Cranial base surgery has made tremendous advancements over the past decade due to innovative developments in preoperative imaging and intraoperative monitoring. Skull base surgery, including reconstructive procedures, can be challenging even for skilled neurosurgeons because of the lack of surgical space. It is challenging for reconstructive surgeons to select the type of reconstruction that lowers the risk of major complications, such as

\section{Correspondence: Jong Won Hong}

Department of Plastic and Reconstructive Surgery, Institute for Human Tissue

Restoration, Severance Hospital, Yonsei University College of Medicine,

50-1 Yonsei-ro, Seodaemun-gu, Seoul 03722, Korea

E-mail: saturn@yuhs.ac

This study was supported by a faculty research grant from the Yonsei University College of Medicine (No. 6-2016-0147).

Received July 16, 2021 / Revised October 20, 2021 / Accepted October 20, 2021 cerebrospinal fluid leakage, meningitis, abscess, seizure, and even death $[1,2]$. Therefore, a watertight dural seal and vascularized coverage to support neural and vascular structures from the extracranial oral and nasal cavities are primary considerations.

Local flaps, distant pedicled fasciocutaneous flaps, and muscle flaps (e.g., galeal and temporalis muscle flaps) have traditionally provided vascularized tissue for dural seals [3-5]. The choice of a reconstructive technique depends on multiple factors (e.g., defect size and the involvement of an irradiated field). In recent years, skull base reconstruction surgery has undergone significant changes because of technological and operative advances. However, reconstruction is challenging if tumor invades the olfactory bulb, frontal sinus, or orbital wall extending from the nasal cavity to the intracranial area. In such cases, free vascularized flaps are considered the gold standard for larger defects $[4,6]$. This technique provides adequate tissue bulk as required 
with a reliably abundant blood supply that promotes wound healing and reduces the risk of significant postoperative complications such as cerebrospinal fluid leakage, meningitis, and pneumocephalus $[2,4,6,7]$. In this study, we successfully reconstructed large anterior cranial base defects that involve the intracranial and nasal cavity using the anterolateral thigh (ALT) free flap. Therefore, we suggest that an ALT free flap is a suitable and versatile surgical option to reconstruct large anterior and inner cranial base defects.

\section{METHODS}

\section{Patients}

Five male patients (age range, 50-75 years) from 2018 to 2021 were enrolled in this study (IRB No. 4-2021-0658). These patients underwent anterior skull base surgery with reconstruction using an ALT free flap. The patients were diagnosed with transitional meningioma, ameloblastoma, olfactory neuroblastoma, adenocarcinoma, and squamous cell carcinoma. The tumor was of intracranial origin in one case and of extracranial origin in the remaining four cases. In three cases, the patients underwent preoperative radiotherapy with exposure of the superior temporal artery (STA) and superior temporal vein (STV) (Table 1). Tumor excision was done by neurosurgeons in cooperation with otorhinolaryngologists. Reconstruction surgery was performed by the plastic surgery team. Retrospective charts and radiologic images of these patients were reviewed.

\section{Surgical method}

To use the STA safely in reconstructive surgery, preparing the vessel should be performed first. The STA path was traced using a handheld Doppler device and marked preoperatively. Pinning was performed after the induction of general anesthesia, and the neurosurgeon was consulted to ensure that pinning was not performed around the STA. The plastic surgery team participated in pinning and confirmed the location of the STA. Since the STA lies between the galeal and subcutaneous layers, dissection before scalp flap elevation is critical to avoid vessel injury. We designed a bicoronal incision along the direction of the STA.

The first incision was made over the superficial temporal vessels in order to secure them (Fig. 1A). The surgeon should consider that the STV usually, but not always, runs alongside the STA. The frontal branch of the STA is sometimes dissected, but this branch is usually less suitable than the parietal branch because it is thinner and runs through the forehead. Therefore, the area near the zygomatic arch should be dissected before STA bifurcation. Because tumor resection is time-consuming, the dissected vessels should not be clipped to avoid blood clots. In addition, moist conditions were used to prevent desiccation.

When designing the flap with the defect volume in mind it was positioned more distally on the thigh in order to obtain a longer pedicle length (Fig. 1B). The medial incision was made first, and pedicle dissection was performed simultaneously with the excision of the tumor (Fig. 1C). Thorough skin de-epithelialization is followed before flap elevation because the flap lie in the intracranial space (Fig. 1D). Preserving as much tissue (e.g., fat and fascia) as possible is recommended because the narrow and deep space of the intracranial space to the nasal cavity makes it challenging to estimate the exact volume to reconstruct. If there is unnecessary tissue, it may be removed during reconstructive surgery.

Because the flap cannot be inset and fixed inside the intracranial area, the space should be filled as much as possible to prevent the creation of dead space. To reduce volume loss due to postoperative muscle shrinkage, inclusion of muscle was kept to a minimum. The flap was used in a folded manner to prevent it from being pushed upwards (Fig. 2). This technique obstructs the area from the frontal sinus and nasal cavity (Fig 3 ).

Vascular anastomosis was performed after insetting the flap to prevent tension resulting from flap repositioning during the insetting process (Fig. 1E). After anastomosis, we considered burring the resected frontal bone at the point where flap could be pressed. Frontal bone fixation was then performed by the plastic surgery team (Fig. 1F). In particular, in cases where the defect size is small to moderate, local or regional flaps, such as pericranial flaps, are usually satisfactory.

Table 1. Summary of patient information

\begin{tabular}{|c|c|c|c|c|c|c|c|c|c|c|}
\hline $\begin{array}{l}\text { Case } \\
\text { no. }\end{array}$ & $\begin{array}{l}\text { Age } \\
\text { (yr) }\end{array}$ & Sex & Diagnosis & Previous therapy & $\begin{array}{l}\text { Primary tumor } \\
\text { site }\end{array}$ & $\begin{array}{l}\text { Flap size } \\
\left(\mathrm{cm}^{2}\right)\end{array}$ & $\begin{array}{l}\text { Pedicle length } \\
\text { (cm) }\end{array}$ & $\begin{array}{c}\text { Site of } \\
\text { anastomosis }\end{array}$ & $\begin{array}{c}\text { Postoperative } \\
\text { therapy }\end{array}$ & Operation time \\
\hline 1 & 51 & Male & Adenocarcinoma & None & Nasal cavity & $21 \times 9.5$ & 8.2 & Left STA, STV & Radiotherapy & $9 \mathrm{hr}$ \\
\hline 2 & 50 & Male & Transitional meningioma & None & Intracranial area & $18 \times 6$ & 9 & Right STA, STV & Radiotherapy & 16 hr 50 min \\
\hline 3 & 75 & Male & Ameloblastoma & Radio therapy & Nasal cavity & $20 \times 7$ & 10 & Left STA, STV & Radiotherapy & $11 \mathrm{hr}$ \\
\hline 4 & 52 & Male & Olfactory blastoma & Radio therapy & Frontal sinus & $14 \times 6$ & 8.3 & Left STA, STV & None & $14 \mathrm{hr} 36 \mathrm{~min}$ \\
\hline 5 & 69 & Male & Squamous cell carcinoma & Radio therapy & $\begin{array}{l}\text { Ethmoid sinus, } \\
\text { frontal sinus }\end{array}$ & $15 \times 6.5$ & 9.2 & Right STA, STV & None & $15 \mathrm{hr} 31 \mathrm{~min}$ \\
\hline
\end{tabular}

STA, superior temporal artery; STV, superior temporal vein. 

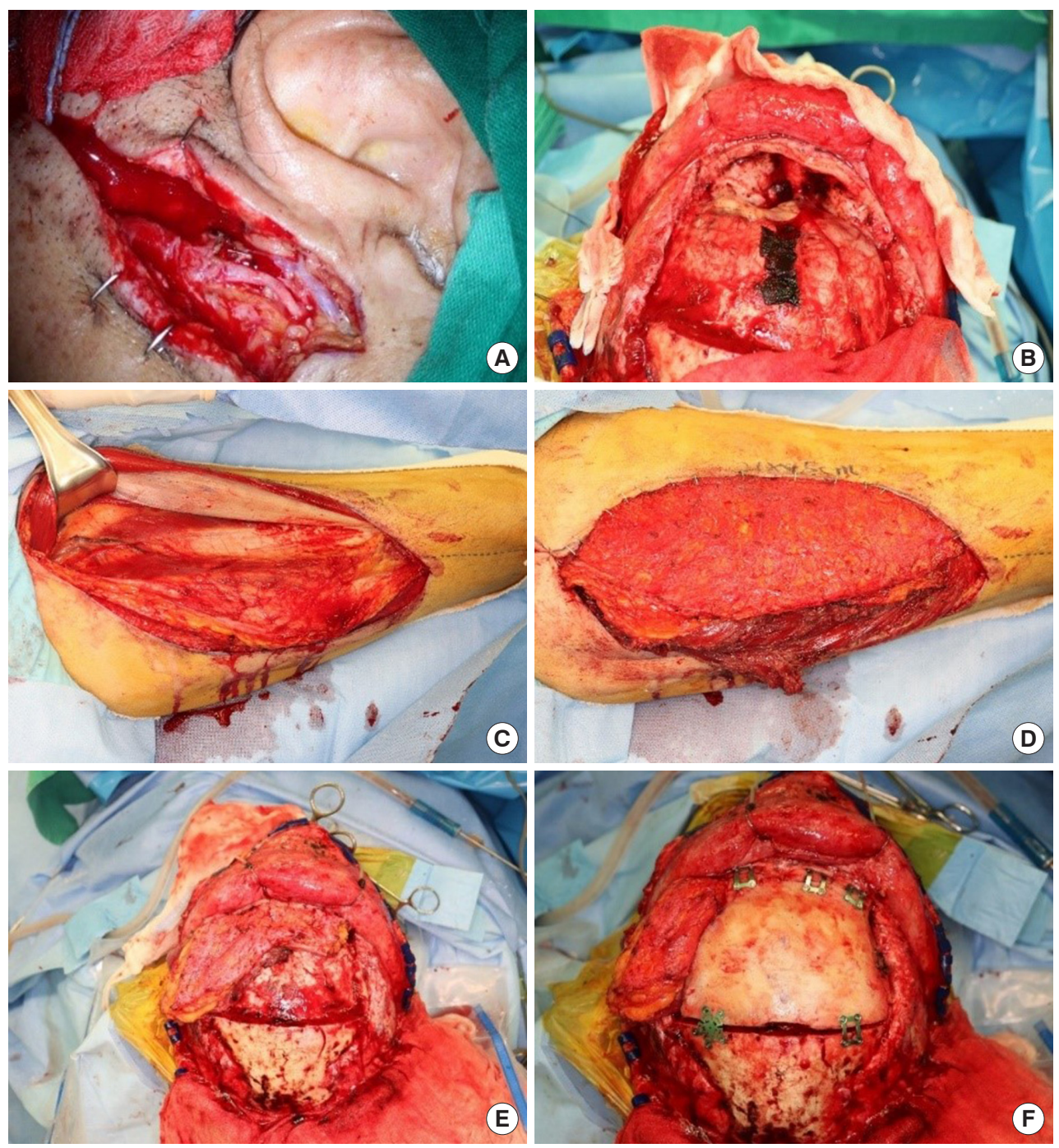

Fig. 1. Preoperative and intraoperative photographs of case no. 1, a 51-year-old man who underwent anterior skull base reconstruction using an anterolateral thigh (ALT) free flap. The patient had an anterior skull base defect resulting from a craniotomy and the removal of an adenocarcinoma. (A) Dissection of the superficial temporal artery before a bicoronal incision. (B) Anterior skull base defects, including the frontal sinus and cribriform plate of the ethmoid bone after tumor removal with communication between the nasal cavity and intracranial space. (C) ALT flap elevation with vessel dissection simultaneously performed via the upper incision during tumor resection. (D) De-epithelialization of the ALT flap. (E) The ALT flap is inset to line the defect. (F) Frontal bone fixation to avoid interference with the inset flap.
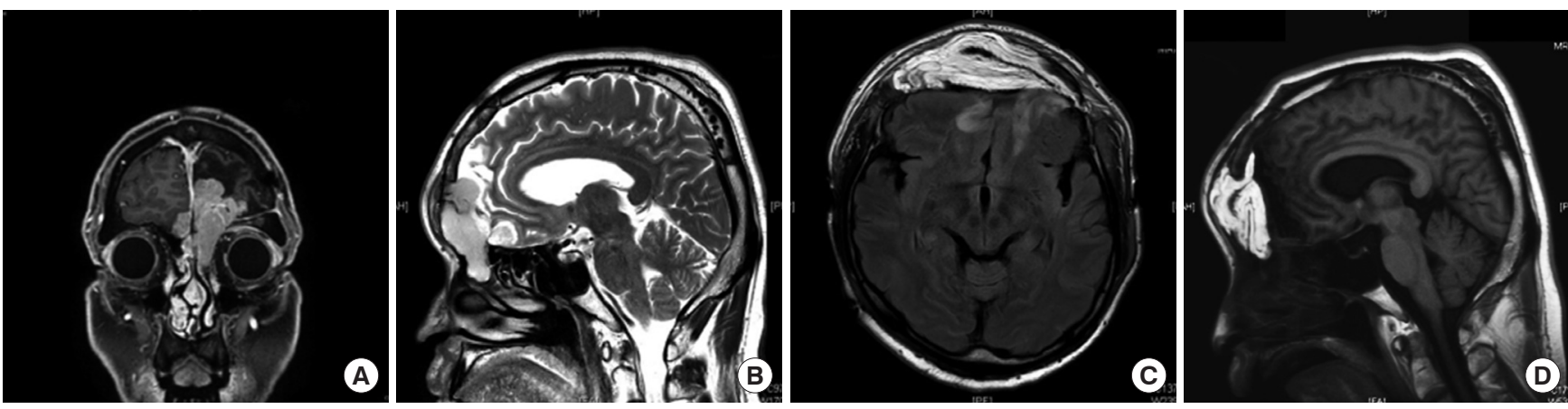

Fig. 2. Magnetic resonance images (MRI) of case no. 1. (A, B) Preoperative MRI: an approximately 7-cm enhancing hemorrhagic mass in the left nasal cavity, with invasion to the left frontal lobe and left superior orbit. (C, D) Postoperative MRI: the anterolateral thigh flap is well maintained 1 month postoperatively, and the defect between the intracranial space and nasal cavity is repaired with the folded flap. (A, D) T1weighted images. (B, C) T2-weighted images. 

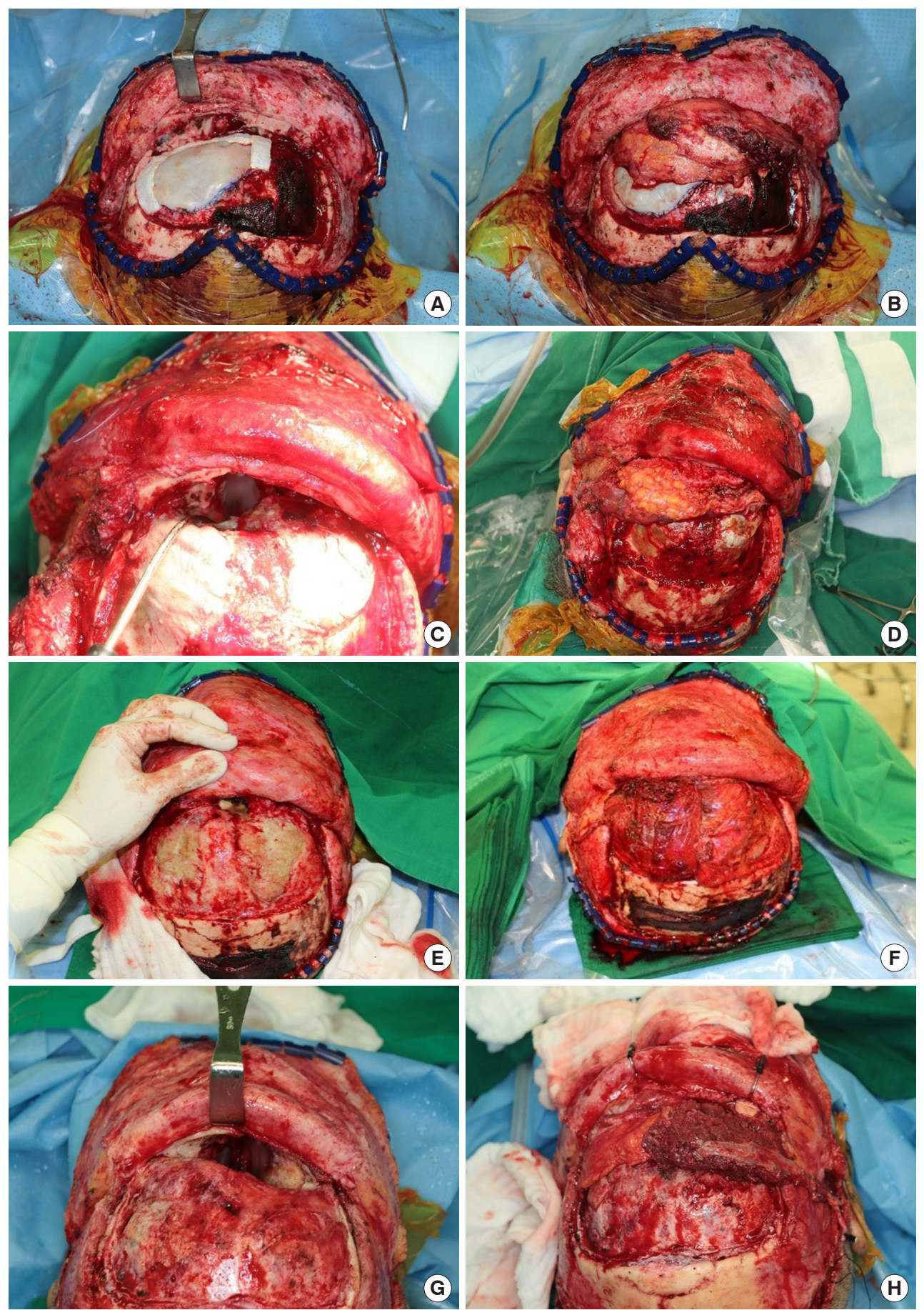

Fig. 3. Intraoperative photographs of case nos. 2, 3, 4, and 5. (A, C, E, G) An extensive anterior skull base defect after tumor removal with communication between the nasal cavity and intracranial space. (B, D, F, H) An anterolateral thigh flap inset to line the defect.

After surgery, we placed a continuous suction system using a Hemovac line inserted into the nasal cavity to drain the remaining irrigation solution or postoperative secretions. This method was the same as that previously described for the reconstruction of head and neck tumors [8]. Patients were routinely admitted to the intensive care unit postoperatively for monitoring and ventilatory support. It was recommended to maintain the head elevation to $30^{\circ}$ after surgery to prevent discharge from ascending to the intracranial area. Mechanical ventilation was weaned on the 1st postoperative day.

Absolute bed rest was ensured for 1 week after surgery until the continuous suction catheter placed in the nasal cavity was removed. Meanwhile, $30^{\circ}$ head elevation was maintained for at least 2 weeks. Three days of absolute nil-per-os status were nec- 
essary because of the possible requirement for reoperation at any time. Thereafter, sips of water were gradually allowed. Starting 1 week postoperatively, L-tube feeding was implemented to avoid coughing. Since it is impossible to monitor the flap directly, we periodically checked for signs of inflammation such as spiking fever and drainage in the Hemovac. One week postoperatively, the otorhinolaryngology team endoscopically checked whether the skull base had been successfully sealed. Magnetic resonance imaging (MRI) was also conducted 1 month postoperatively.

\section{RESULTS}

Five patients underwent the removal of anterior skull base tumors for reconstruction with an ALT free flap. The defects involved an open frontal sinus and communication with the nasal cavity. The average size of the flaps was $125.7 \mathrm{~cm}^{2}$ while the mean operation time was 13 hours and 23 minutes. The total reconstruction time required for the plastic surgery team was difficult to calculate because its participation was intermittent during recipient vessel dissection, flap elevation, and reconstructive surgery.

All surgeries were successful without any complications. After surgery, no infections or suspicious turbid changes in the Hemovac were observed. Although flap survival could not be directly confirmed, indirect evidence showed that the flaps all remained in good condition after skull base reconstruction. Postoperative MRI showed no fat necrosis, and a sufficient flap volume and barrier between the intracranial and nasal cavities were observed (Fig. 2C and D).

\section{DISCUSSION}

The anterior skull base, comprising the base of the anterior cranial fossa, is close to the paranasal sinuses and nasopharynx, which can be a source of infection. Its narrow and deep space and the thin basal dura tightly attached to the bone make reconstruction of the skull base challenging. In an ideal skull base reconstruction procedure, a reconstructive surgeon should conduct watertight sealing (to prevent cerebrospinal fluid leakage), separate the nasocranial spaces to secure a sufficient volume to fill the dead space, and use well-vascularized tissue to cover the defect [9-11].

The last decade has witnessed the emergence of new options for anterior skull base reconstruction due to improvements in microvascular techniques [12], which has made it possible to perform more radical tumor extirpation. However, failure of anterior skull base reconstruction can cause major complica- tions such as fatal infections due to nasocranial communication, cerebrospinal fluid leakage, tension pneumocephalus, seizure, and meningocele [12-14]. For small or moderate size defects local or regional flaps, such as pericranial flaps, are usually satisfactory [15-17]. However, to reconstruct more extensive anterior skull base defects and prevent intracranial-nasopharyngeal communication through a watertight seal, a reliable flap with named vascular supply (e.g., a bilateral reverse temporalis muscle flap) is considered an excellent choice. This flap is frequently used because it has a low incidence of failure due to the absence of vessel anastomosis and induces minimal donor site morbidity. Additionally, because the bilateral temporalis muscle is connected to the intracranial area, this flap does not require specific fixation $[18,19]$. Our institution used a reverse temporalis muscle flap for the reconstruction of anterior skull base defects. To reinforce the skull base, we additionally raised a galeal flap combined with a reverse temporalis muscle flap [19].

Despite the positive outcomes described above, this local technique has some drawbacks: (1) local flaps, such as the pericranial flap and reverse temporalis flap, cannot reliably separate and seal the brain from the bacterial flora of the upper airway in patients with previous high-dose radiation therapy or requiring extensive resection of the cranial base [2]; (2) osteotomy must be performed on both sides of the frontal bone to place a bilateral reverse temporalis muscle flap in the intracranial area, but this procedure provokes aesthetic complaints (e.g., depression or temporal hollowing); and (3) and prevention of intracranial complications with local flaps compared to the free flaps have shown inferior result, which may be due to the distal portion of the local flap that is placed at the most critical portion of the reconstruction having the most precarious blood supply [20].

Therefore, our institution has recently selected free tissue transfer as the treatment of choice in these cases, carefully considering patients' circumstances (e.g., previous radiation therapy or plans for postoperative radiotherapy). The choice of free flap may vary depending on the surgeon's preference: the flap should contain as much soft tissue as possible with a long pedicle, and free flaps are preferred for the treatment of irradiated defects [19]. Many donor sites have been described in the literature $[21,22]$, including the ALT, rectus abdominis myocutaneous, and latissimus dorsi free flaps.

A rectus abdominis free flap can be harvested without changing the patient's position. This flap is also advantageous because it is a myofascial free flap with substantial volume, based on the deep inferior epigastric artery, a sufficiently long pedicle. However, this approach also has limitations, such as the risk of hernia at the donor site, muscle atrophy after reconstruction, significant postoperative pain, and variation in the harvested vol- 
ume due to adipose tissue [1].

The next most commonly used flap is the latissimus dorsi free flap, which provides sufficient reconstruction volume and pedicle length. However, it also has disadvantages such as the inability to raise the flap while performing a tumor resection, muscle atrophy leading to dead space in the recipient site, and donor site morbidity. After comparing these various methods, we selected the ALT free flap as the first choice for reconstruction. The ALT flap is an excellent option for reconstructing anterior skull base defects due to its versatility, pedicle reliability, and minimal donor site morbidity without requiring an intraoperative position change [23]. To avoid muscle atrophy, we harvested the ALT free flap mainly with fat tissue.

Some muscle tissue was included along the perforator during pedicle dissection. Although we did not include much muscle tissue to fill the volume, a watertight seal was well secured postoperatively with the harvested volume. Thorough de-epithelialization was performed with the dermal plexus intact because it was impossible to specify the exact location of flap fixation in advance. ALT flap elevation was performed simultaneously with tumor excision, without altering the patient's position. Once the defect size was determined after tumor excision, the other half of the flap outline was redesigned and further dissected. This strategy saves time and makes it easier for surgeons to maintain their focus.

The margin of the bone defect should be trimmed by burring the bony spurs to prevent irritation and the creation of dead space after insetting the flap, instead of immediately placing the flap after completing the tumor resection. Furthermore, the position where the pedicle will be placed should be refined with burring to avoid kinking and pressure after closing the scalp. After trimming the space, massive irrigation should be performed using Yankauer suction, including the nasal cavity, to prevent ascending infection. The procedure is then followed by bone fixation, Hemovac line tip, and scalp suture. A surgeon who specializes in reconstructive surgery should also perform these procedures if a neurosurgeon has not been working with the surgical team for a long time.

The dermal side is usually considered resistant to infection, but it has not been established whether to reconstruct the nasal side with the fascial side or the de-epithelized dermal side, and we did not specify which surface would face the nasal cavity. The flap surface facing the open nasal cavity was expected to undergo secondary healing; to promote this, we administered antihistamines and encouraged postoperative head elevation.

Intraoperatively managing each surgical field is critical; the vascular pedicle should be checked during neurosurgery, and the brain parenchyma should be monitored during plastic sur- gery. In particular, these areas should be maintained in a wet environment to prevent desiccation, and irrigation and consistent monitoring are necessary. It is also recommended to insert an L-tube preoperatively to avoid misdirection toward the intracranial area if the nasal approach is not required. If the preinserted L-tube is likely to be disrupted during otorhinolaryngologic surgery, it is acceptable to insert it immediately before reconstruction.

After surgery, ascending infection of the nasal cavity should be prevented. To avoid this complication and create an environment favorable for drying, we used a continuous irrigation and suction system that was usually kept in place for 7 days postoperatively. The head's elevation should be maintained at $30^{\circ}$ to prevent any postoperative discharge from ascending.

In this study, successful reconstruction was performed using ALT free flaps for large anterior skull base defects or when a reverse temporalis muscle flap could not be used. The reverse temporalis muscle flap is also a good choice, particularly if technical and psychological difficulties hinder free vascularized flap transfer. A prerequisite for performing this procedure was to form a reconstructive surgery team with experience in free flaps. If reconstruction using the reverse temporalis muscle flap fails, conversion to a free flap using the proximal part of the STA and STV is the second option. However, it should be noted that if the free flap is attempted first and fails, it is possible to perform anastomosis using the STA and STV on the contralateral side or the facial artery and vein. However, this may require a longer pedicle or additional vein grafts. Considering these complications, successfully performing the first free flap is critical, while thorough preparation for the second option should be considered in advance.

\section{NOTES}

\section{Conflict of interest}

No potential conflict of interest relevant to this article was reported.

\section{Ethical approval}

The study was approved by the Institutional Review Board of Severance Hospital, Yonsei University Health System (IRB No. 4-2021-0658) and performed in accordance with the principles of the Declaration of Helsinki. The informed consent was waived because this study design is a retrospective chart review.

\section{Patient consent}

The patients provided written informed consent for the publication and the use of their images. 


\section{ORCID}

Shin Hyun Kim

Won Jai Lee

Jong Hee Chang

Joo Hyung Moon

Seok Gu Kang

Chang Hoon Kim

Jong Won Hong

\section{Author contribution}

Conceptualization: Jong Won Hong. Data curation: Shin Hyun Kim, Jong Hee Chang, Joo Hyung Moon, Seok Gu Kang, Chang Hoon Kim, Jong Won Hong, Won Jai Lee. Formal analysis: Shin Hyun Kim, Jong Won Hong. Funding acquisition: Jong Won Hong. Methodology: Shin Hyun Kim, Jong Won Hong. Project administration: Jong Won Hong. Visualization: Shin Hyun Kim. Writing - original draft: Shin Hyun Kim. Writing - review \& editing: Won Jai Lee, Jong Hee Chang, Joo Hyung Moon, Seok Gu Kang, Chang Hoon Kim, Jong Won Hong. All authors read and approved the final manuscript.

\section{REFERENCES}

1. Gullane PJ, Lipa JE, Novak CB, Neligan PC. Reconstruction of skull base defects. Clin Plast Surg 2005;32:391-9.

2. Chang DW, Langstein HN, Gupta A, De Monte F, Do KA, Wang X, et al. Reconstructive management of cranial base defects after tumor ablation. Plast Reconstr Surg 2001;107:134657.

3. Menderes A, Yilmaz M, Vayvada H, Demirdover C, Barutcu A. Reverse temporalis muscle flap for the reconstruction of orbital exenteration defects. Ann Plast Surg 2002;48:521-6.

4. Kwon SG, Kim YO, Rah DK. Anterior cranial base reconstruction with a reverse temporalis muscle flap and calvarial bone graft. Arch Plast Surg 2012;39:345-51.

5. Chung SW, Hong JW, Lee WJ, Kim YO. Extended temporalis flap for skull base reconstruction. Arch Craniofac Surg 2019; 20:126-9.

6. Valentini V, Fabiani F, Nicolai G, Torroni A, Gennaro P, Marianetti TM, et al. Use of microvascular free flaps in the reconstruction of the anterior and middle skull base. J Craniofac Surg 2006;17:790-6.

7. Cantu G, Solero CL, Pizzi N, Nardo L, Mattavelli F. Skull base reconstruction after anterior craniofacial resection. J Craniomaxillofac Surg 1999;27:228-34.

8. Chang HP, Hong JW, Lee WJ, Kim YS, Koh YW, Kim SH, et al. Incorporating a continuous suction system as a preventive measure against fistula-related complications in head and neck reconstructive surgery. Arch Plast Surg 2018;45:449-57.

9. Gil Z, Abergel A, Leider-Trejo L, Khafif A, Margalit N, Amir A, et al. A comprehensive algorithm for anterior skull base reconstruction after oncological resections. Skull Base 2007;17:25-37.

10. Girod A, Boissonnet H, Jouffroy T, Rodriguez J. Latissimus dorsi free flap reconstruction of anterior skull base defects. J Craniomaxillofac Surg 2012;40:177-9.

11. Hanasono MM, Silva A, Skoracki RJ, Gidley PW, DeMonte F, Hanna EY, et al. Skull base reconstruction: an updated approach. Plast Reconstr Surg 2011;128:675-86.

12. Schmalbach CE, Webb DE, Weitzel EK. Anterior skull base reconstruction: a review of current techniques. Curr Opin Otolaryngol Head Neck Surg 2010;18:238-43.

13. Boyle JO, Shah KC, Shah JP. Craniofacial resection for malignant neoplasms of the skull base: an overview. J Surg Oncol 1998;69:275-84.

14. Gagliardi F, Boari N, Mortini P. Reconstruction techniques in skull base surgery. J Craniofac Surg 2011;22:1015-20.

15. Langstein HN, Chang DW, Robb GL. Coverage of skull base defects. Clin Plast Surg 2001;28:375-87.

16. Noone MC, Osguthorpe JD, Patel S. Pericranial flap for closure of paramedian anterior skull base defects. Otolaryngol Head Neck Surg 2002;127:494-500.

17. Kim YW, Lee DH, Cheon YW. Secondary reconstruction of frontal sinus fracture. Arch Craniofac Surg 2016;17:103-10.

18. Kim YO, Park BY. Reverse temporalis muscle flap: treatment of large anterior cranial base defect with direct intracranial-nasopharyngeal communication. Plast Reconstr Surg 1995;96:57684.

19. Shin D, Yang CE, Kim YO, Hong JW, Lee WJ, Lew DH, et al. Huge anterior skull base defect reconstruction on communicating between cranium and nasal cavity: combination flap of galeal flap and reverse temporalis flap. J Craniofac Surg 2020; 31:436-9.

20. Neligan PC, Mulholland S, Irish J, Gullane PJ, Boyd JB, Gentili F, et al. Flap selection in cranial base reconstruction. Plast Reconstr Surg 1996;98:1159-68.

21. Amin A, Rifaat M, Civantos F, Weed D, Abu-Sedira M, Bassiouny M. Free anterolateral thigh flap for reconstruction of major craniofacial defects. J Reconstr Microsurg 2006;22:97104.

22. Malata CM, Tehrani H, Kumiponjera D, Hardy DG, Moffat DA. Use of anterolateral thigh and lateral arm fasciocutaneous free flaps in lateral skull base reconstruction. Ann Plast Surg 2006;57:169-76.

23. Suh JM, Chung CH, Chang YJ. Head and neck reconstruction using free flaps: a 30-year medical record review. Arch Craniofac Surg 2021;22:38-44. 